\title{
ANGELAH: A Framework for Assisting Elders At Home
}

\author{
Tarik Taleb ${ }^{1, *}$, Dario Bottazzi ${ }^{2, \dagger}$, Mohsen Guizani ${ }^{3, \ddagger}$, and Hammadi Nait-Charif ${ }^{4, \S}$ \\ ${ }^{1}$ Graduate School of Information Sciences, Tohoku University, Japan \\ ${ }^{2}$ Department of Electronics, Computer Science, and System Engineering, University of Bologna, Italy \\ ${ }^{3}$ Department of Computer Science, Western Michigan University, USA \\ ${ }^{4}$ National Centre for Computer Animation, Bournemouth University, UK \\ * talebtarik@ieee.org, ${ }^{\dagger}$ dario.bottazzi@unibo.it, ${ }^{\ddagger}$ mguizani@ieee.org, ${ }^{\S}$ hncharif@ bournemouth.ac.uk
}

\begin{abstract}
The ever growing percentage of elderly people within modern societies poses welfare systems under relevant stress. In fact, partial and progressive loss of motor, sensorial, and/or cognitive skills renders elders unable to live autonomously, eventually leading to their hospitalization. This results in both relevant emotional and economic costs.

Ubiquitous computing technologies can offer interesting opportunities for in-house safety and autonomy. However, existing systems partially address in-house safety requirements and typically focus on only elder monitoring and emergency detection. The paper presents ANGELAH, a middleware-level solution integrating both "elder monitoring and emergency detection" solutions and networking solutions. ANGELAH has two main features: $i$ ) it enables efficient integration between a variety of sensors and actuators deployed at home for emergency detection and $i i$ ) provides a solid framework for creating and managing rescue teams composed of individuals willing to promptly assist elders in case of emergency situations. A prototype of ANGELAH, designed for a case study for helping elders with vision impairments, is developed and interesting results are obtained from both computer simulations and a real-network testbed.
\end{abstract}

Index Terms-Ubiquitous assistance, pervasive computing, healthcare network, and elderly assistance.

\section{INTRODUCTION}

Major developed-world societies are experiencing a frightening demographic phenomenon of a rapid growth of their aging population [1]. As the elderly people percentage is mounting, health and social costs are increasing too, thus putting welfare systems under relevant stress. In fact, progressive degradation of vision, hearing, motion, and cognitive skills deprives people from the possibility of independently performing basic activities, such as, self-care, leisure, household, and social interactions, and often forces elders' hospitalization, with both emotional and economic notable impacts.

Technological advances and cost reduction in computing devices and network solutions can play an important role in enhancing elderly people independence. In particular, recent developments in wireless technologies, sensors, and actuators are enabling new classes of eldercare applications available anywhere and at anytime, i.e., ubiquitous eldercare services. The common guideline behind ubiquitous eldercare is the complete shift of the locus of health control from hospitals to pervasive systems deployed close to where elderly users live and move, with the main goals of increased independence, safety, and quality of life on one hand, and of care cost-saving on the other hand. Along this line, several ubiquitous eldercare solutions have recently appeared in the literature that permit to record and analyze elders behavioral patterns, to monitor seniors' mobility, to assist individuals in daily activities, such as self-care, and so forth.

However, to the best of our knowledge, only few solutions aim at addressing domestic safety for elders [2], [3]. Individuals safety is a prerequisite for autonomous life-styles. The increase, each year in deaths and injuries, especially among elders, has shown up "in-house safety" as an emergent field of research. In fact, everyday tasks are a continuous source of danger for elders due to their eventual decline in physical and cognitive skills.

Supporting in-house safety is a rather challenging task that requires suitable answers to several research questions:

- how can we monitor and detect possibly dangerous situations?

- how can we design unobtrusive assistive solutions that facilitate integration, management and update of sensors and actuators infrastructure within elders home environments?

- how can we improve emergency detection and response?

- and finally, how can we identify suitable trade-offs that keep into account all of these considerations?

The main motivation behind this research work emerges from the conviction that the graceful integration of Commercial Off-The-Shelf (COTS) devices and networking elements, along with pervasive computing technologies, can offer significant opportunities to provide safety and quality of life to elderly in need of help. This research work presents the ANGELAH (AssistiNG ELders At Home) framework, a middleware-level solution able of $i$ ) integrating sensors and actuators needed to monitor and guarantee elder safety, ii) detecting possibly dangerous situations for the elder, and iii) composing emergency response groups of volunteers and caregivers, allocated in the nearby, willing to help in case of an emergency event [3]. As will be explained later, the emergency response group members are chosen according to several criteria, such as their physical proximity to elders home and their medical skills, following the multi-attribute decision making (MADM) theory [4].

The remainder of this paper is organized as follows. Section 
II sets the present work within the state of the art. Section III identifies the major requirements and design guidelines that should be followed to support in-house elder safety services. Section IV presents the ANGELAH model. Section $\mathrm{V}$ portrays the system architecture. Section VI shows relevant implementation insights on response group member selection. Section VII presents a case study of the proposed ANGELAH framework. Concluding remarks follow in Section VIII.

\section{RELATED RESEARCH WORK}

To better position the past and on-going research activities about ubiquitous eldercare, let us first introduce a solution classification widely accepted in the medical area. The US National Center on Medical Rehabilitation Research (NCMRR) has proposed a model for categorizing the research and development efforts in technology for aging in well conditions [5]. The NCMRR model identifies five main working directions at different levels, each focusing on a specific problem: cellular, organ, action, task-role, and social limitations. The cellular level relates to aberrations in normal physiological processes and in the cellular structure, with the consequent diseases and/or genetic abnormalities. The organ level focuses on solutions to impairments of organs, e.g., the heart, or of whole organ systems, e.g., the cardio-vascular system, while the action level refers to person inabilities to properly perform some actions due to functional limitations of the responsible organs. The research at the task-role level is addressed to people disabilities in properly performing tasks and activities in specific physical and social contexts. The highest research area in the NCMRR model is about social barriers that obstacle people engagement in a rich socio-emotional environment.

Each NCMRR level involves specific technological requirements and calls for proper solutions to enable elder autonomy in daily living, with less dependencies on family, friends, and caregivers support. Let us note that only in the cellular area the research focus is more on cure and recovery of aging people rather than on technological solutions to improve their quality of life. More in details, research efforts in the organ and action levels seek to design and develop devices to compensate limitations in common physical functionality, such as movement or hearing. For example, advanced signal processing technologies have permitted to significantly improve the sound quality provided via digital hearing aids. Technologies at the NCMRR task-role level aim at reducing the impact of disabilities into the aging people life. For instance, a hearing aid should also allow aging people with hearing disabilities to actively participate in usual conversations. Research efforts in the social limitation level, instead, analyze the social attitudes of aging people to derive the guidelines for the development of technological solutions that can improve the social interactions of elders with the external world.

Pervasive computing offers relevant and challenging opportunities to design and implement ubiquitous eldercare solutions at the task-role and social limitation levels [23]. In fact, on one hand, ubiquitous assistance solutions enable the realization of wearable devices with ubiquitous connectivity that can operate at the task-role level by assisting elders in their activities and by addressing their disabilities anywhere and anytime. On the other hand, ubiquitous care networking supports permit to overcome social limitations by providing elders with a plethora of communication artifacts and services specifically tailored to set them within the context of a rich social and emotional framework and to reduce their sense of loneliness.

Ubiquitous assistance solutions. In the first category, there has been a plethora of research work addressing various aspects of telemedicine [6]. The scope of these researches range from standardization activities for telemedicine deployment [7], privacy and security solutions [8] to realization of a system [9], [10], [11]. The main concerns of these solutions consist in assisting elders in their routine life activities, constant monitoring of their health conditions, and prompt alerting in emergency events. In [12], a computer-vision based system is proposed to support people with severe vision impairments. To secure a safe navigation of a particular environment, the system generates alert messages, via a speech output interface, whenever a change occurs to the layout of the environment. This makes people, with vision deficiency and living in that environment, aware of the occurring changes. In [13], artificial intelligence learning and planning techniques are used to define proper steps of basic activities of daily living (e.g., hand-washing). The resultant system provides visual or verbal instructions to a person with dementia on how to perform a particular daily living activity. The system consists of three modules; a tracking module which uses computer vision to monitor the actions of the user by determining the spatial coordinates of the person's body and hands within the environment. Once these coordinates have been determined, a planning module determines what step the user is completing and whether the step being completed is correct. If the system detects that the user has made an error, such as completing a step out of sequence or missing a step altogether, the prompting module selects and plays a prompt message. In [14], a cognitive orthotic system, called auto-minder, is proposed. The system models the daily plans of an individual and decides on when and where to remind the person of the execution of those plans. The developed techniques are deployed on a mobile robot, as part of the Nursebot project's Initiative on Personal Robotic Assistance of the Elderly. Benny et al., in their UbiSense system, used embedded smart vision techniques to detect changes in posture, gait and activities. In addition to monitoring normal daily activities and detecting potential adverse events such as falls, the system aims to capture signs of deterioration of the patients by analyzing subtle changes in posture and gait [15]. In their later work, they used image sensing and visionbased reasoning to verify and further analyze events reported by other sensors such as Accelerometer for fall detection [16]. The Honeywell Laboratories' Independent Life Style Assistant (ILSA) is another notable example of integrated smart environments which aim at ubiquitous assistance [17]. In the ILSA system, multiple JADE agents are deployed. The agents support data monitoring via home-installed sensors. The collected data are aggregated and processed to make adequate response planning and machine learning. UbiMedic [18] and Code Blue [19] are other notable agent-based smart home assistance systems. A general observation about the different 
industrial and academic proposals of ubiquitous assistance is that they focus on only specific tasks, and thus provide only a subset of the support functions needed for eldercare assistance.

Ubiquitous care networking. The ubiquitous care networking area is still in its infancy, with only a few solution proposals. The primary objective of ubiquitous care networking solutions is to promote social interactions of elders with their surroundings and to coordinate between the different actors involved in a ubiquitous eldercare event. In [20], a context-aware middleware solution, dubbed Allocation and Group Aware Pervasive Environment (AGAPE), is proposed for the creation and management of ad-hoc assistance teams to provide outdoor emergency assistance to senior citizens in need of immediate help.

To conclude, different solutions have been devised in the recent literature for both ubiquitous assistance and ubiquitous care networking support. To the best knowledge of the authors, no solution in the literature has integrated the two types of ubiquitous eldercare into one single solution. This challenging task underpins the focus of the research work outlined in this paper.

\section{Ubiquitous In-House SAFETy REQuiREMENTS AND DESIGN GUIDELINES}

The design of technological solutions for ubiquitous inhouse safety is a rather challenging problem. Most of available works in the literature provide ad-hoc solutions for elder monitoring, provide tools to detect specific emergency situations for the elder, and typically provide basic support for alerting health authorities.

Elder monitoring is typically performed by exploiting sensors, such as, RFIDs, accelerometers, pressure sensors, videocameras that are pervasively deployed in the environment where the elder lives and/or embedded in the devices he ${ }^{1}$ currently uses. However, most available research work typically focuses on specific elder pathologies and can only partially address the requirements stemming from in-house safety. Several technological challenges have to be addressed to support elder monitoring. In fact, notwithstanding the widespread availability of low-cost sensors of different types, sensors currently exhibit a high heterogeneity in nature, accuracy, and performance and should be chosen according to elders' needs and pathology. They should be unobtrusive and must not alter the existing living environment; their monitored data should be as much interoperable as possible, so that different assistance tasks can exploit the context information deriving from the same sensors; they might be changed during the time according to the progressive changes in elders' needs [21], [22], [23]. In addition, sensor technologies for eldercare should not place any additional load on final users by requiring explicit input/effort from older adults [24]. Indeed, even remembering to wear a device/sensor may represent a cognitive load that a fraction of elder population cannot tolerate, e.g., elderly people affected by dementia.

In case of emergency situations, available in-house safety supports typically permit to alert only heath authorities, and

\footnotetext{
${ }^{1}$ In this paper, the term "he" also refers to she.
}

rarely friends or relatives. However, neighbors and passing-by people may play an important role in emergency situations. Indeed, prompt assistance may become the determining factor between life and death in case of heart attacks: circulation and ventilation should be initiated as soon as possible to avoid irreversible cerebral damage. However, high levels of stress and lack of time, typically characterizing emergency situations, could create untrained and unrehearsed reactions that cause critical delays in intervention and contribute to inconsistencies/omissions in the critical data to share. Novel communication/coordination solutions should proactively propose and stimulate collaboration among potential helpers colocated with the elder in need of help: they should clearly notify helper community members about the occurring situation, should inform helpers about the activities to perform by supporting them in the collaborative definition and assignment of assistance tasks, and should coordinate their activities via synchronization and data sharing, dynamically tailored to the group of participants and to the degree of criticality of the elder situation [3].

According to the above considerations, we need to consider two main design principles when designing an in-house safety support solution: context-awareness and group-based collaboration.

Context-Awareness. The full visibility of the elder context information [25], such as elder physical location, gestures, health status, and clinical record, permits to dynamically determine whether the elder is in need of help. If so, to tailor service provisioning according to the current operating conditions. For instance, in the case of an accidental fall the system can detect the occurred event and may accordingly react by contacting elder's relatives and caregivers, by triggering actuators to unlock the elder's home doors. In addition, the visibility of context information may also permit to tailor the interaction between the elder and the system, e.g., by adopting speechbased user interfaces in the case of individuals with strong visual impairments.

Context information should be obtained by sensor infrastructures composed on the basis of elder needs and pathologies. As a consequence, context information tend to show a high degree of heterogeneity, making it necessary to carefully consider mechanisms and tools to turn raw monitoring data from sensors into context information at a higher level of abstraction, ready to be used by the application level. In particular, to improve software re-usability and to accelerate in-house safety service customization, it is necessary to decouple the design and implementation of context aggregation solutions from the development and deployment of assistance services. This permits to hide assistance services from the technological details and difficulties of sensing and monitoring. For example, an emergency response service generally requires knowing when an elder is calling for prompt help, e.g., when he has fallen, rather than the sampled value from a position sensor. In addition, to recognize that a complex context situation has occurred, it is often necessary to compare sensed values from multiple sensors. Aggregation is the support functionality that allows interpreting and reasoning on complex context situations by putting together and processing the raw monitoring 
data coming from sensors, generally increasing the abstraction level of context data.

Many-to-many context data distribution is another important requirement to take into account in the design of ubiquitous assistance services. On one hand, assistance applications need to exploit context that may come from different, and often heterogeneous, sources. On the other hand, the same context information should be provided to several distributed service components, thus enabling different ubiquitous eldercare services to obtain the needed context information from the same sensor infrastructure. For instance, an integrated positioning system may consist of indoor (Wi-Fi-based) and outdoor (GPS-based) location detectors, possibly producing positioning data in different formats. The result of their work should be distributed to both an emergency service and an elder-tracking service for family members.

Group-based Collaboration. Grouping is the primary issue to address in the design of support mechanisms and tools for in-house safety. The metaphor of group, team, or community can facilitate interactions among individuals. In fact, the group abstraction permits to restrict the scope of in-house safety information dissemination/sharing, such as emergency alerts, to only the members of a group. This can significantly simplify the establishment of inter-member interactions and decision making. Various types of eldercare communities can be formed spontaneously to provide impromptu assistance without any prior planning. In fact, group membership management for inhouse safety scenarios cannot rely on preconceived knowledge about the set of available group members, their characteristics, and their device properties. On the contrary, it is necessary to provide users with the possibility to compose, dissolve, join, and leave groups on-demand from highly heterogeneous terminals, with possibly different forms of wireless connectivity. For instance, teams consisting of individuals in physical proximity with an elder in faint can be dynamically created to provide prompt assistance to the elder. These groups may include people with limited medical skills, professional caregivers, as well as doctors who are currently located in physical proximity.

Context awareness is emerging as a crucial property in the design of group management solutions for in-house safety. Novel group supports should exploit the full visibility of context information, such as elder conditions, location of users/terminals/resources, user profile of preferences and skills, to promote the collaboration among members willing to assist the elder in-house anytime. Among the various and heterogeneous data belonging to context, location plays a primary role. For instance, group management supports could exploit the visibility of elder location to create an eldercare support group on the fly, composed of only the entities in the elder vicinity to provide prompt help. This may be particularly relevant in the case of emergency situations, such as heart attacks, where response operations are subject to severe time constraints. The visibility of further context information, such as individuals' medical skills, may also guide the formation of suitable ad-hoc groups: for example, medical skills may permit to preferably compose response groups of people with appropriate experience to cope with the emergency situation.
In the proposed ANGELAH framework, different context factors are envisioned and MADM theory is applied to them to form adequate groups of volunteers.

\section{THE ANGELAH MOdEL}

Fig. 1 depicts the ANGELAH system model. The peculiarity of ANGELAH is the full integration between sensing and monitoring technologies used to detect possibly dangerous situations for the elder, along with mobile groupware collaboration supports that enable coordination among responders willing to engage in elder assistance. ANGELAH identifies different management roles with different responsibilities: the Sensing Entity (SE) role, the Actuator Entity (AE) role, the Home Manager (HM) role, the Surveillance Center (SC) role, the Locality Manager (LM) role, and finally the Local Responder (LR) role.

SEs represent sensors that are deployed over the elder's living environment. SEs continuously gather row context data and communicate them to the HM. As depicted in Fig. 1, several SEs are deployed in elders homes, and different sensing technologies may be applied to monitor elder behavior and physical conditions according to his actual needs (e.g., elder's location, heart-beat, environment temperature, etc).

AEs represent actuators disseminated within the elder's apartment. AEs are controlled by the HM. They enforce appropriate actions that permit smooth monitoring of elders conditions. For example, when the elder enters a room, RFID tag reader (AE) turns on camera sensors and other SEs to monitor the elder's movement and behavior (Fig. 1).

The HM operates as a central server and is in charge of gathering available context information from deployed SEs, aggregating this information, and detecting whether the elder is in need of help. According to the current situation, HM also triggers the execution of safety-related actions that typically involve the coordination among different AEs (e.g., to close possibly open gas knobs). When an emergency situation is detected, the HM notifies the SC of the event via an emergency notification message, referred to as SOS throughout this paper, followed by some context information that may help a SC agent to define the emergency level and type, its causes, and the kind of assistance the elder may be in need of (Fig. 2).

LRs represent individuals willing to provide prompt help to elders when an emergency situation occurs. LRs are statically subscribed to the ANGELAH service. They can be family members of the elder, his friends, relatives living in his immediate surroundings, or simply passers-by, neighborhood community representative and paid help, such as professional caregivers, doctors, pharmacists, etc. Each LR is characterized by a unique User IDentifier (UID), and a profile describing his characteristics, such as user identity, user's current physical location, user's medical expertise, user's history record and skills in providing assistance within the ANGELAH framework, and the trust SC associates with him/her. LRs' devices have features that enable them to discover, join and leave elder support groups, to obtain the visibility of available partners allocated in the nearby, along with their profile information, and to collaborate with them via message exchange. 


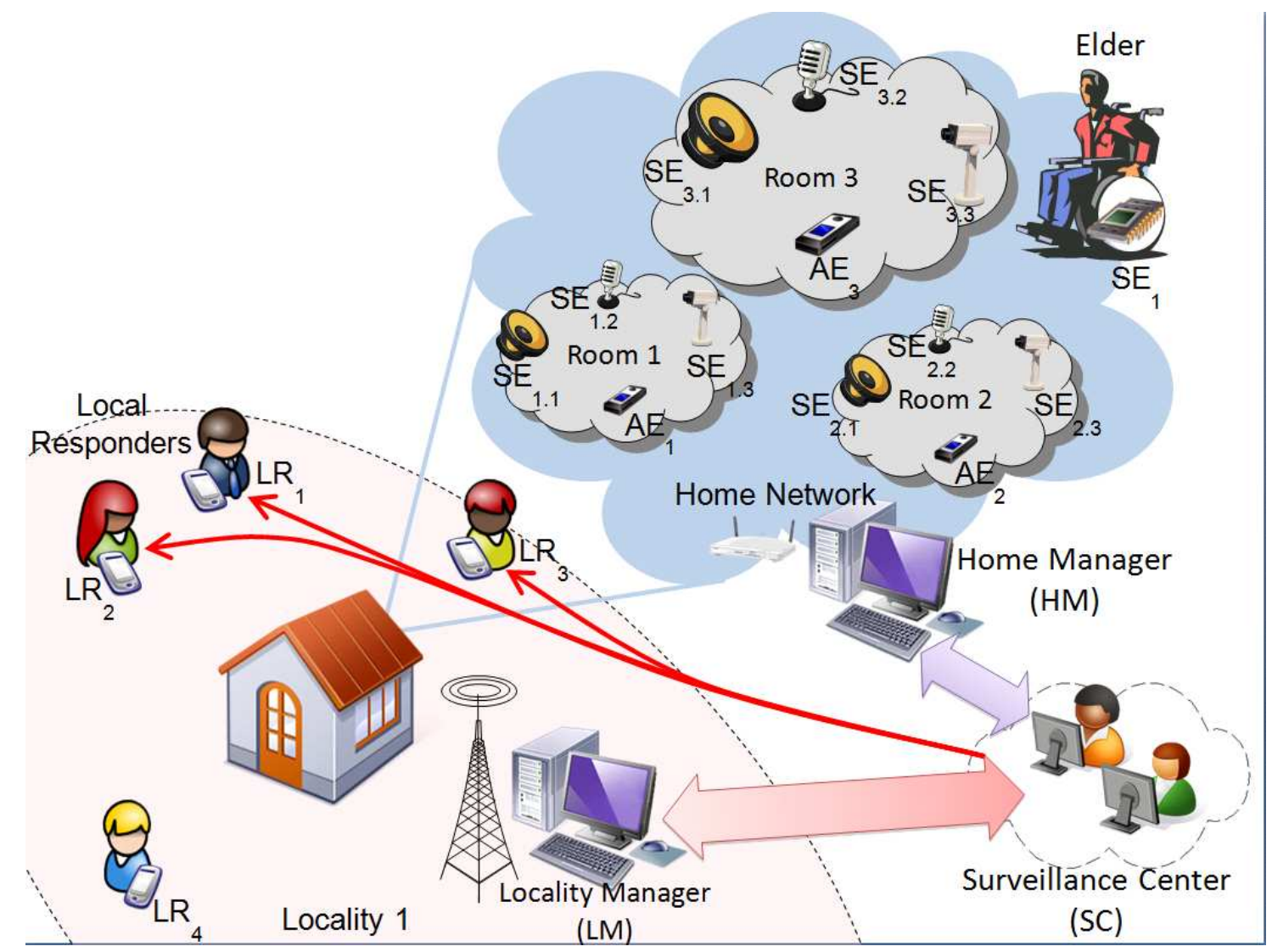

Fig. 1. The ANGELAH model.

SC is in charge of coordinating prompt response in emergency situations. When SC receives a SOS message from an elder's HM, it first obtains the visibility of all LRs allocated in the proximity of the elder's home. Then, on the basis of the visibility of LR's profile information, SC promotes the formation of a support group composed of the best suited LRs willing to assist the elder. Fig. 2 shows the sequence of messages exchanged between the SC and LRs. Upon receiving a SOS message, the SC defines the emergency type from the context information analysis and broadcasts a "Call For Assistance - CFA" message to LRs currently in the proximity of the elder. CFA messages may include information such as personal information of the senior (e.g., age, gender, etc), the postal address of his residence, his physical and cognitive characteristics, the kind of assistance he is in need of, along with additional information (if available) describing the current conditions of the elder (e.g., pulse). In response to the CFA message, LRs willing to help send back an "Acceptance Notification - AN" message to the SC. These reply messages contain personal information of the volunteers (e.g., UID), their current location, and the estimated time it may take them to get to the location of the elder in need of help. SC then runs an algorithm to select the most adequate LRs. Once adequate LRs are sorted out, SC notifies them and provides them with information on how to access the elder's residence and with instructions on how to assist him/her.

It is worth noticing that composing a response group is a rather difficult problem that call for the identification of tradeoff solutions between multiple contrasting principles, such as LR's physical location and distance with regards to the elder's home, his medical skills, the elder pathologies and so forth, that require to solve MADM problems. Once the SC selects a set of adequate individuals willing to provide assistance to the elder, it notifies them and provides them with instructions on how to assist the elder. In ANGELAH, each elder support group is uniquely identified by a Group IDentifier (GID) and a profile that includes information on the elder's identity, his pathologies, his contact information and home address, and so forth.

ANGELAH is based on the locality concept both to mitigate the complexity of the group formation problem and to reduce responders' intervention time. ANGELAH defines a locality as the set of all LRs located within the same network cell. ANGELAH statically associates a LM to each locality, which is in charge of monitoring the availability of collocated LRs. It is worth noticing that several elders, possibly needing assistance may be residing in the same network locality. In addition, due to wireless network deployment it is also possible for different localities to partially overlap. LRs can freely roam between different localities and may belong to more than one locality at any time. Locality also plays an important role in responders group management, because it limits the group management scope. In fact, each group can be allocated only within a single locality. However, a locality can host several 
elder support groups aiming at providing assistance to different elders.

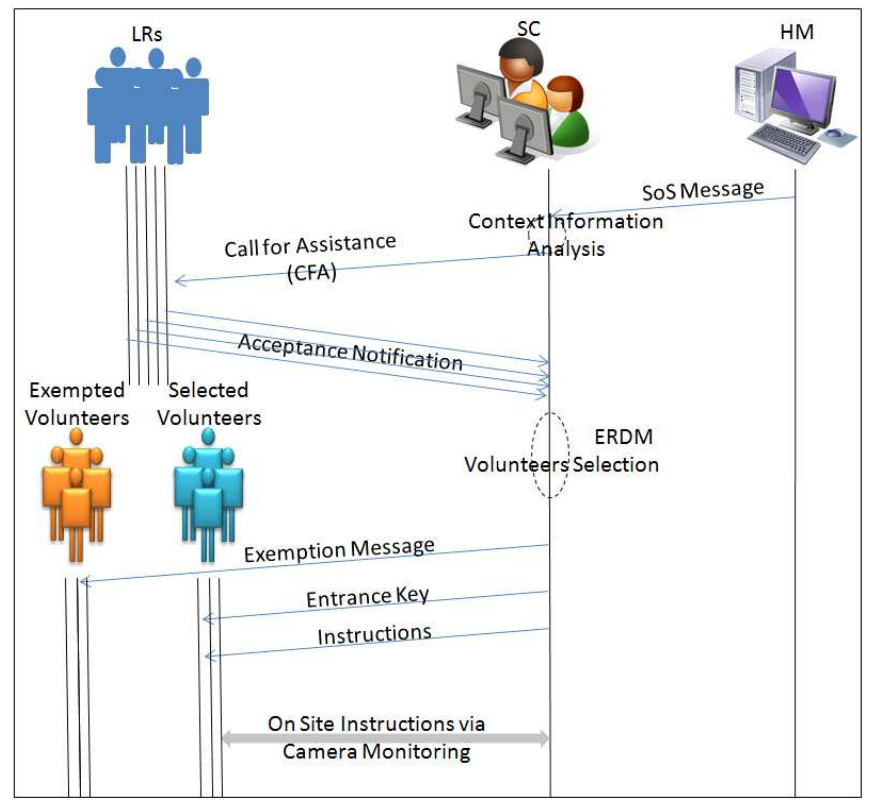

Fig. 2. Sequence of signaling messages exchanged between HM, SC, and LRs.

\section{The ANGELAH ARChitecture}

Fig. 3 portrays the ANGELAH middleware architecture implemented on top of the Java virtual machine. As Fig. 3 depicts, the layered ANGELAH architecture is mainly composed of three layers: the Response Management layer, the Monitoring and Assistance layer, and the Group Collaboration layer.

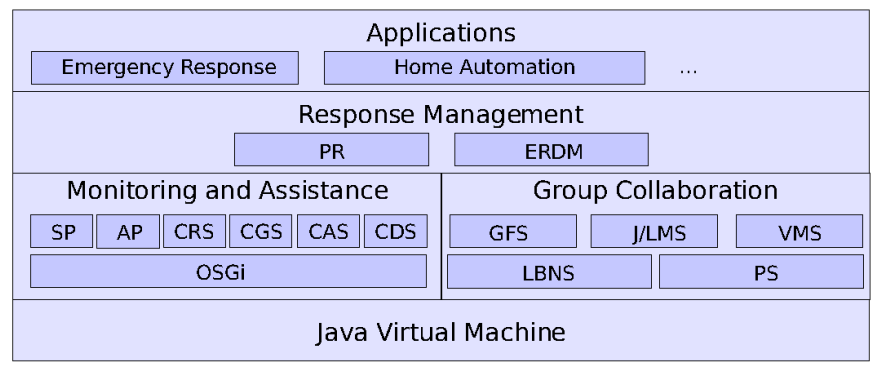

SP: Sensor Proxy AP: Actuator Proxy CRS: Context Repository Service CGS: Context Gathering Service CAS: Context Aggregation Servic CDS: Context Distribution Service

PR: Profile Repository ERDM: Emergency Response Decision Making GFS: Group Formation Service J/LMS: Join/Leave Manager Sen

LBNS: Location Based Naming Service PS: Proximity Service

Fig. 3. The ANGELAH middleware architecture.

\section{A. Monitoring and Assistance Layer}

The monitoring and assistance layer is in charge of $i$ ) integrating and managing all available sensors and actuators deployed in the elder's home; ii) gathering, aggregating, and distributing all sensed context information; iii) detecting whether the elder needs assistance; and finally $i v$ ) controlling

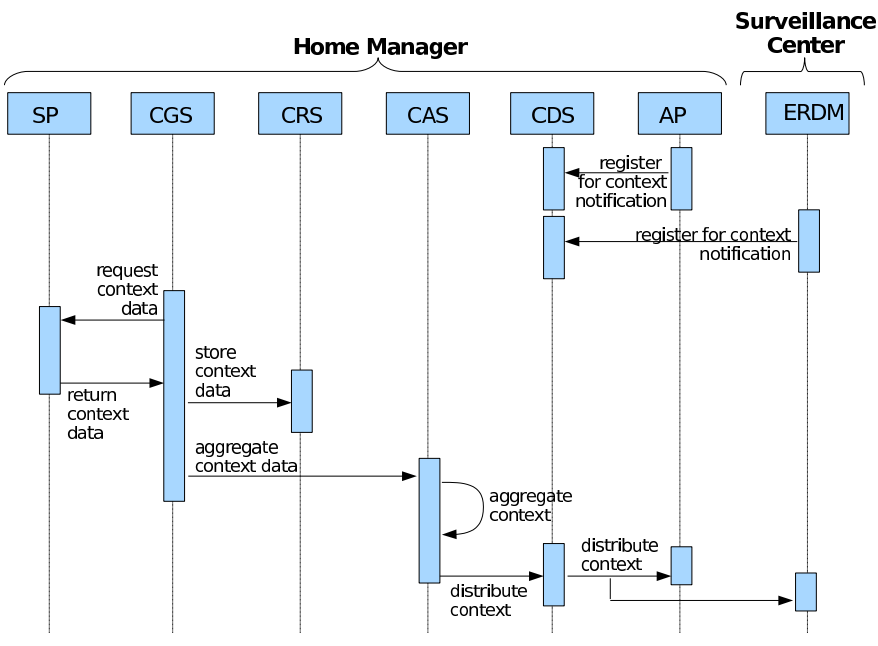

Fig. 4. Monitoring and assistance layer service interaction diagram.

available actuators and alerting the surveillance center in case of an emergency situation.

All monitoring and assistance layer support facilities are developed on top of the Open Service Gateway initiative (OSGi) infrastructure. In fact, broad consensus has been reached in the smart home research community on the important features of OSGi to easily integrate and manage heterogeneous home appliances. The peculiarity of OSGi is to provide a serviceoriented, component-based support that significantly simplifies software lifecycle management and provides the required support to compose and frequently update sensor/actuator infrastructures according to the evolving elder needs.

The major interactions between the monitoring and assistance layer's components are portrayed in Fig. 4. At regular times, the Context Gathering Service (CGS) requests sensed data from the available sensors. The time between consecutive requests hinges on sensor technical characteristics. For example, a location sensor might be frequently sampled to obtain a precise snapshot of elder location, whereas a temperature sensor can be sampled less frequently (e.g., every 10 minutes) due to the large amount of time needed to change elder's home temperature. In ANGELAH, each sensor is statically associated to a Sensor Proxy (SP). SP is juxtaposed between the sensors and the ANGELAH context management facilities; thus decoupling the context gathering logic from sensor-specific technical details and simplifying the context information collecting process. Once the current sensed data are obtained from the available SEs, CGS forwards the obtained values to both Context Repository Service (CRS) and Context Aggregation Service (CAS). CRS persistently maintains context information that is required to build-up and update elder behavioral models that form the basis to detect possibly dangerous situations and to facilitate diagnosis of elder's pathologies. On the other hand, CAS aggregates context information obtained from different context sources and detects possibly dangerous situations. Finally, Context Distribution Service (CDS) is in charge of distributing the aggregated context data to the interested entities. For example, in case of an emergency, CDS notifies a SOS message to the 
Emergency Response Decision Making (ERDM) instance installed at SC, including information required for the formation of an elder support group, such as elder's home address, elder's identity, and emergency type.

It should be emphasized here that while it is possible to consider a self-functioning approach where upon emergency detection, HM wirelessly broadcasts SOS messages to an adhoc of passers-by, with no involvement of SC similar in spirit to the outdoor emergency assistance system proposed in [20], such an approach gives rise to two major issues, namely safety and false alerts. The first issue is highly important as we are aiming at indoor environments and the second is due to the fact that any robust risk detection technique, which may be installed in CAS, may still have limitations in accurately capturing an elder's activities and fully understanding his behavior, based on which anomaly can be detected. Involvement of personnel of a central surveillance center in confirming the emergency occurrence and detecting its causes and type is of high importance.

\section{B. Response Management Layer}

Upon reception of an emergency notification message (e.g., SOS message) from an elder's HM, the response management layer triggers the ERDM instance to form groups composed of individuals willing to provide assistance to the elder. The main interactions generated at the response management layer are depicted in Fig. 5. As shown in the figure, ERDM coordinates with the Proximity Service, installed at the LM of the locality where the elder resides, and requests the visibility of all nearby LRs (e.g., their UID) willing to provide prompt assistance to the elder. By coordinating with the Profile Repository (PR) at SC, ERDM can obtain further information on available helpers, such as their medical expertise, their history record and skills in providing assistance within the ANGELAH framework, and the trust SC associates with them. On the basis of available information, ERDM solves a MADM problem to compose a response group formed from the most adequate individuals willing to help the elder. Then, ERDM coordinates with the Group Formation Service (GFS) for promoting the newly formed group and inviting all selected individuals to join it.

\section{Group Collaboration Layer}

The group collaboration layer evolves from the AGAPE middleware [20]. It provides the basic functions to compose, dissolve, and manage emergency response groups in wireless environments.

When an emergency occurs, the GFS instance, installed at LM, promotes the creation of a new response group. For this purpose, GFS coordinates with the Location-Based Naming Service (LBNS) that randomly generates and assigns GIDs (and PIDs) by exploiting the Universally Unique Identifier (UUID) naming approach (Fig. 6). GFS also requests ERDM for the elder profile information (e.g., elder's identity, address, pathologies, emergency level, etc.) and for the list of selected helpers that should form the new emergency response group. Following this, GFS coordinates with the Join/Leave Manager

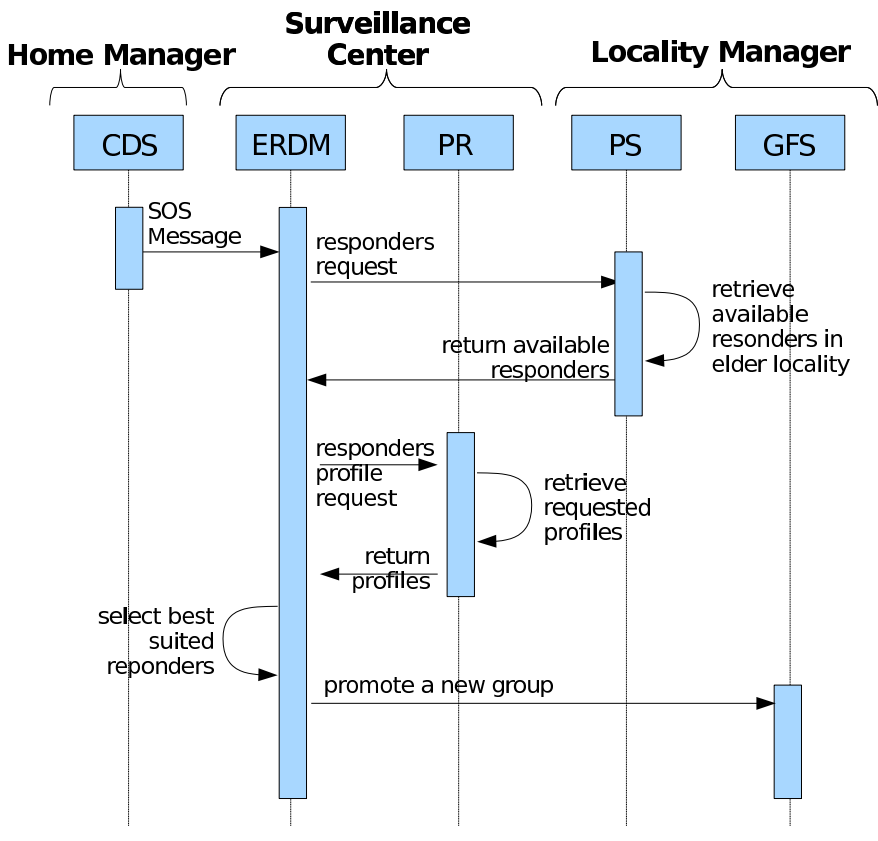

Fig. 5. Response management layer service interaction diagram.

Service (J/LMS) installed at helpers' devices to invite them to join the group.

At regular times, the View Manager Service (VMS) creates, maintains, and disseminates views to ANGELAH group members. A view contains the list of collocated group members along with their profiles (context-dependent view). Each view entry includes several data: group member PID/IP address obtained from PS, member's identity and medical skills obtained from PR. In addition, to cope with mobility-induced changes in group membership, VMS coordinates with PS for the notification of arrival, departure and disconnection of a group member entity, and accordingly updates views. It is worth stressing out that the PS instance, installed at LM, monitors for responders' availability using periodic advertisement messages from LRs. When the lag between consecutive advertisement messages from a LR exceeds a threshold value, the LR is assumed to be disconnected. It is also worth noting that the threshold value between consecutive advertisements should be chosen according to different criteria, such as the average number of responders in a locality, their mobility behavior, the locality surface, and so on.

\section{INSIGHTS FOR ERDM IMPLEMENTATION}

This section presents insights on the MADM algorithm used by the ERDM instance at SC to select adequate volunteers among a group of LRs to cope with a particular emergency situation. Before delving into details, we list up the used notations:

- $M$ : number of emergency levels.

- $\Im_{i}$ : emergency level $i(i \in\{1,2, \cdots, M\})$.

- $\theta_{\alpha . i}$ : action time for emergency level $\Im_{i}$.

- $\tau_{i}$ : timeout for waiting for AN messages from LRs in case of an emergency level $\Im_{i}$.

- $\gamma_{i}$ : LR selection threshold associated with the emergency level $\Im_{i}$. 
Location Manager

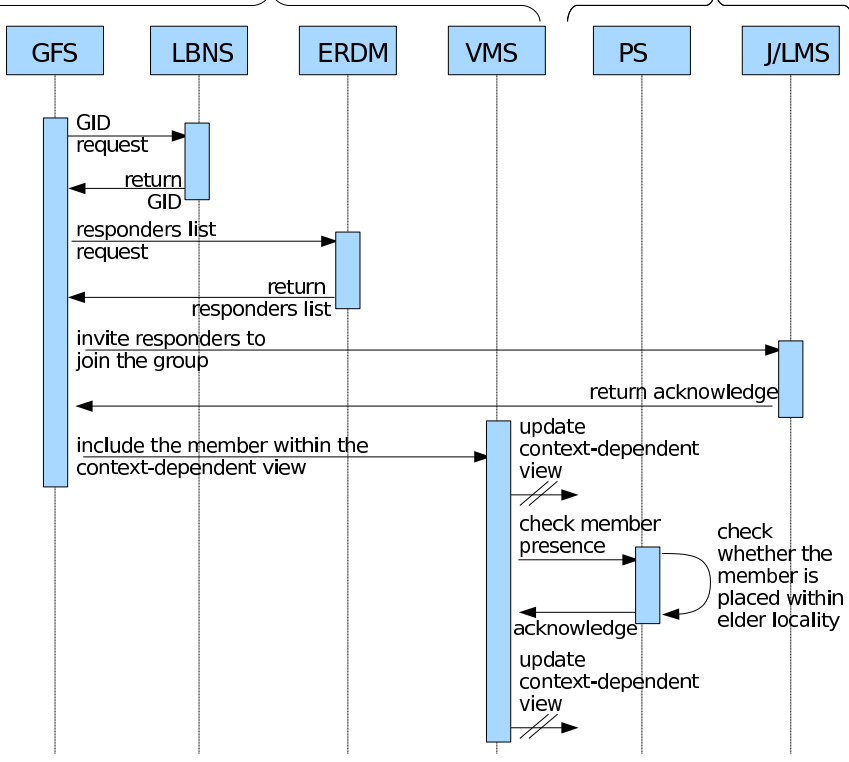

Fig. 6. Group collaboration layer service interaction diagram.

- $N_{i}^{S}$ : required number of skillful LRs for emergency level $\Im_{i}$.

- $N_{i}^{T}$ : total number of required LRs (regardless of their skills) for emergency level $\Im_{i}$.

- $\ell$ : number of attributes associated with LRs.

- $X_{k, j}: j^{t h}$ attribute of LR $S_{k}(j \in\{1,2, \cdots, \ell\})$.

- $A_{k}$ : vector of attributes of LR $S_{k} \quad\left(A_{k}=\right.$ $\left.\left[X_{k, 1}, X_{k, 2} \cdots, X_{k, \ell}\right]\right)$.

- $\omega_{i, j}$ : weight associated with the $j^{t h}$ attribute in case of emergency level $\Im_{i}$.

- $\Omega_{i}$ : vector of weights associated with emergency level $\Im_{i}$ $\left(\Omega_{i}=\left[\omega_{i, 1}, \omega_{i, 2} \cdots, \omega_{i, \ell}\right]\right)$.

As stated earlier, the LR selection mechanism is initiated by ERDM based on the MADM theory. Indeed, in ANGELAH, the PR instance at SC maintains profiles of each LR; already subscribed to the ANGELAH service. For each subscriber $S_{k}$, a set of attributes $\left\{X_{k, j}, j \in\{1,2, \cdots, \ell\}\right.$ is associated. The attributes represent $i$ ) the expertise and skills of LRs, $i i$ ) their history record in providing assistance, and iii) the trust level SC associates with them. These attributes are constantly updated and maintained by SC. Table I shows a typical format of the profile table SC maintains on registered LRs.

TABLE I

A TYPICAL FORMAT OF LRS PROFILE.

\begin{tabular}{|c|c|c|c|c|}
\hline $\begin{array}{c}\text { LR ID } \\
S_{k}\end{array}$ & $\begin{array}{c}1^{\text {st }} \text { Attribute } \\
X_{1}\end{array}$ & $\begin{array}{c}2^{\text {nd }} \text { Attribute } \\
X_{2}\end{array}$ & $\cdots$ & $\ell^{t h}$ Attribute \\
$X_{\ell}$
\end{tabular}

In ANGELAH, we assume that there are $M$ emergency levels defined a priori at the surveillance center. For each emergency level $\Im_{i}(i \in\{1,2, \cdots, M\})$ and each attribute
$X_{j}(j \in\{1,2, \cdots, \ell\})$, SC defines a weight $\omega_{i, j}$ as shown in Table II. Additionally, with each emergency level $\Im_{i}$, three parameters are associated:

- action time $\theta_{\alpha . i}$ : minimum time within which assistance should be provided to the elder.

- waiting timeout $\tau_{i}$ : maximum time SC should wait for to receive AN messages from LRs.

- acceptance threshold $\gamma_{i}$ : threshold for selecting LRs.

These three parameters should be carefully set by the surveillance center. For example, in case of a life-threatening event (e.g., heart attack), both action time $\theta_{\alpha . i}$ and waiting timeout $\tau_{i}$ should be set to small values. In case of a bone fracture due to a fall, expertise and skills become more important so the system can set $\gamma$ to high values with particular focus on the skills-related attribute. Action time $\theta_{\alpha}$ and timeout $\tau$ can be set to relatively high values.

Upon receiving a SOS message, the agent in charge (at SC) first defines the corresponding emergency level based on $i$ ) the event type (e.g., fall, faintness, heart-attack) determinable from the context information sent by the CDS instance (e.g., capture video) and $i i$ ) the profile of the senior (e.g., physical and cognitive characteristics) available at the PR instance. Let $\Im_{m}$ denote the selected emergency level. For a timeout $\tau_{m}$, ERDM waits for responses from LRs. Once the system receives the required $N_{m}^{S}$ or $N_{m}^{T}$ replies, or the timeout $\tau_{m}$ expires, ERDM sorts out the LRs based on information available in their AN messages, such as their physical proximity and availability, using the action time $\Theta_{\alpha . m}$ and following an analysis as will be explained later at the end of this section. Out of the sorted LRs, those with attributes satisfying the following condition are chosen.

$$
A_{k} \cdot \Omega_{m}^{T}=\sum_{n=1}^{\ell} X_{k, n} \cdot \omega_{m, n} \geq \gamma_{m}
$$

In case the number of LRs selected at the second phase are numerous, only an adequate number of LRs are requested to assist and are provided with information on how to access the senior's residence along with instructions corresponding to the determined emergency level $\Im_{m}$.

In the remainder of this section, we analyze the system responsiveness and derive, from the analysis, conditions on the required availability of LRs. The defined conditions are used in the selection procedure of LRs. In the analysis, the following notations are used:

- $\theta_{d}$ : time elapsed since the actual occurrence of the event till its detection by CAS at HM. This may also include time required by HM to confirm the anomaly either by setting a timeout or having the senior confirm that he is indeed in need of help.

- $\theta_{S o S}$ : time required to send a SoS message along with the required context information from CDS at HM to ERDM at SC.

- $\theta_{H}$ : time required by the agent to analyze the video and to determine the emergency level. With this regard, agents should be well trained to be able to determine emergency levels within short $\theta_{H}$ times. 
TABLE II

EMERGENCY LEVELS AND THEIR ASSOCIATED PARAMETERS.

\begin{tabular}{|c|c|c|c|c|c|c|c|}
\hline $\begin{array}{c}\text { Emergency } \\
\text { Level }\end{array}$ & $\begin{array}{c}1^{\text {st }} \text { Attribute } \\
X_{1}\end{array}$ & $\begin{array}{c}2^{\text {nd }} \text { Attribute } \\
X_{2}\end{array}$ & $\cdots$ & $\begin{array}{c}\ell^{t h} \text { Attribute } \\
X_{\ell}\end{array}$ & $\begin{array}{c}\text { Action } \\
\text { Time }\end{array}$ & $\begin{array}{c}\text { Acceptance } \\
\text { Threshold }\end{array}$ & $\begin{array}{c}\text { Waiting } \\
\text { Timeout }\end{array}$ \\
\hline \hline$\Im_{1}$ & $\omega_{1.1}$ & $\omega_{1.2}$ & $\cdots$ & $\omega_{1 . \ell}$ & $\theta_{\alpha .1}$ & $\gamma_{1}$ & $\tau_{1}$ \\
\hline$\Im_{2}$ & $\omega_{2.1}$ & $\omega_{2.2}$ & $\cdots$ & $\omega_{2 . \ell}$ & $\theta_{\alpha .2}$ & $\gamma_{2}$ & $\tau_{2}$ \\
\hline$\vdots$ & $\vdots$ & $\vdots$ & $\vdots$ & $\vdots$ & $\vdots$ & $\vdots$ & $\vdots$ \\
\hline$\Im_{M}$ & $\omega_{M .1}$ & $\omega_{M .2}$ & $\cdots$ & $\omega_{M . \ell}$ & $\theta_{\alpha . M}$ & $\gamma_{M}$ & $\tau_{M}$ \\
\hline
\end{tabular}

- $\theta_{E}$ : time required to exchange CFA and AN messages between SC and LRs. This can be equal to the round trip time between the SC and LM.

- $\theta_{E R D M}$ : time required by SC to run the ERDM algorithm for selecting adequate LRs.

As explained earlier, a LR $S_{k}$ notifies the ERDM instance of the time he may need to reach the residence of the senior, should he be selected for the rescue task. Let $\Delta_{k}$ denote the availability of LR $S_{k}$ in terms of time and let $\Im_{m}$ denote the selected emergency level. Using the action time $\Theta_{\alpha . m}$ and the waiting timeout $\tau_{m}$ of the emergency level, LRs with time availability $\Delta_{k}$ satisfying the following inequality are selected.

$\Delta_{k} \leq\left\{\Theta_{\alpha . m}-\tau_{m}-\theta_{d}-\theta_{S o S}-\theta_{H}-\theta_{E}-\theta_{E R D M}\right\}$

The values of $\theta_{d}$ and $\theta_{S o S}$ can be computed by ERDM from the context information sent by CDS.

\section{Case Study: Supporting Elders AfFected by SEVERE VISION IMPAIRMENTS}

To evaluate the performance of the ANGELAH framework in supporting in-house safety for elders, we designed and implemented an application tailored for elders affected by severe vision impairments. Supporting vision impaired individuals is a rather challenging task, as this disability strongly limits elders' free movements and makes individuals more subject to domestic injuries and accidental falls.

\section{A. Deployment Settings}

To secure in-house safety for elders, the underlying sensing, monitoring and positioning technologies should be customized to the actual needs of elders. For in-house location tracking, different techniques have been recently proposed (e.g., intelligent floors and active badges). Whilst these technologies can detect the presence of individuals in a specific location, they either cannot discriminate their identities or, as a remedy to this limitation, require users to constantly hold a device; a solution that is not efficient for seniors affected by dementia.

To cope with this limitation, we consider the use of Radio Frequency IDentification (RFID) technology for elder location tracking. RFID tags are cost-effective and can be adhered to senior clothes. Our envisioned network topology is as depicted in Fig. 1. The actuator and sensor infrastructure is composed of a variety of devices: video cameras, RFID readers, sound sensors, and appliances such as a smart door lock with a password-opening function. Used cameras are standard digital video cameras operating at 30 frames per second, ceilingmounted, with vertically-oriented optical axes, fitted with wide-angle lenses. The position and orientation of the cameras are chosen to minimize occlusion of the elder by furniture. RFID readers are also carefully placed over the house in a way that they provide complete coverage over the entire house. It is worth stressing that, to avoid reader-to-tag interferences, two adjacent readers should be carefully deployed in a way that the difference between their signal strengths is less than the tag's tolerance margin [26]. By functioning as the Reader Network Controller (RNC), HM coordinates among the multiple readers using the EPCglobal Low-Level Reader Protocol (LLRP). To enable elder interaction with the system, microphones and speakers are also placed in all rooms of the elder apartment. These sound I/O interfaces permit the elder to confirm to HM if he is in need of assistance upon an emergency detection by the CAS instance in HM. Intuitively, this operation aims at avoiding false alerts to SC.

A Pentium-based PC running Gentoo Linux, J2SE 1.5, along with all ANGELAH Monitoring and Assistance layer facilities and covering the HM role was also deployed in the apartment. In particular, the system was appropriately configured and enabled to gather context information from the available sensors, i.e., RFID readers, sound sensors and cameras. Each of these entities is statically associated to a particular SP, within the HM, in charge of sampling and processing gathered data. In particular, RFID's SP can both detect and identify elders located in the nearby of the associated reader, whereas camera SP can process the feedback obtained from the associated camera to track the elder, to keep records of his motion, and to make decisions on whether an anomaly occurred in the behavior of the senior [27]. Sound sensors are used to enhance the anomaly detection accuracy of CAS (e.g., in case the monitored senior falls down or screams for help). It is worth noticing that the HM also installs IBM via voice in order to provide elders with a speech based interface.

While this option is not considered in our testbed, for higher accuracy in capturing the elder's behavior, HM needs full visibility of the elder's context, including special layout of the objects in his living environment. Such context awareness is possible by tagging items that are frequently used by the senior (e.g., TV set, Sofa). Whenever a change occurs in the layout of the living environment, updates of the layout is possible via an efficient integration between the inputs from the tag readers and the cameras. The positioning of these items can be also done using technologies such as UWB (Ultra Wide Band) [28]. However these technologies cannot provide accurate indoor positioning and require heavy computation. Moreover, their cost is much higher compared to that of largely affordable RFID tags and readers. 
In our testbed, we also deployed a PC running Gentoo Linux, J2SE 1.5, with all Response Management layer services and playing the $\mathrm{SC}$ role. In addition, by exploiting the wireless Wi-Fi infrastructure deployed in our campus in University of Bologna, we also defined several localities. In our prototype, each network cell defines a locality. In each locality, we have also deployed a server, running Gentoo Linux, J2SE 1.5, playing the LM role and supporting its PS, GFM, and VMS services. Without any loss of generality and for the sake of prototype deployment simplicity, we deployed our prototype on top of the campus-wide Wi-Fi network. In fact, it is still difficult to conduct experimental test-beds on top of communication infrastructures managed and licensed by telecom operators, such as GPRS, UMTS or WiMAX.

Finally, we also setup several wireless-enabled iPAQ PDAs, acting as user terminals for responders. Each device installs Personal Java, J/LMS, and client-side components for PS and VMS. On top of ANGELAH, we also implemented an application prototype that provides available helpers with alert messages when an emergency situation occurs (Fig. 11) and supports message-oriented user collaboration and coordination.

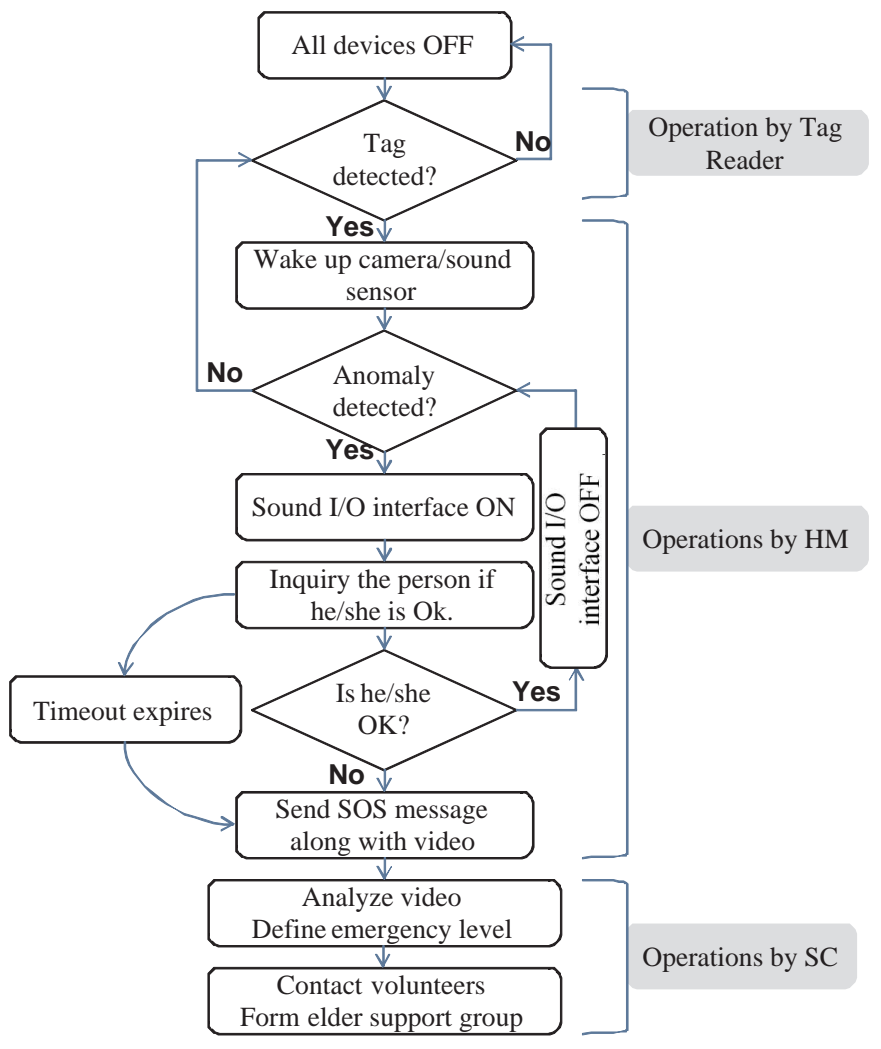

Fig. 7. Major operations considered in the case study.

Fig. 7 depicts the prototype functionality. By exploiting RFID location tracking, elder's HM obtains the visibility of current elder position in his apartment. The visibility of elder's physical location permits to turn on the camera and sound sensors located in the room where he is actually placed. This allows efficient use of energy (i.e., electricity) and permits $\mathrm{HM}$ to analyze only images obtained from a single camera, thus reducing both home network overhead and computational load that may be induced by computer vision techniques. It should be noted that this operation can be also performed using motion sensors. However, in case the senior is sharing the house with other members of the family, the use of motion sensors is not efficient as they cannot distinguish the senior from the other family members. In case of an emergency detection by CAS, the AP application installed over HM turns on the speech-based I/O interface and connects it with microphones and speakers installed in the room where the elder is currently placed. HM first confirms with the senior if there is need for any kind of assistance. Awaiting a response from the person, HM sets up a timeout. In case this is confirmed by the senior or the timeout expires, HM judges the event as an emergency situation and an alert message (SOS) is sent to SC. The audio and video captured from both the camera and sound sensors, respectively, during the time of the emergency event are also sent to SC so that SC personnel may look retrospectively at the moment just before the event occurrence to analyze the cause of the event and to decide whether the person is in an emergency situation and what help the person may be in need of. LRs are contacted and an elder support group is formed from adequate ones. Selected LRs are provided with password to access the house of the senior and the other volunteers are simply exempted and thanked for their eagerness to help. At the same time, the service provider provides the selected volunteers with instructions on how to assist the person. Once the volunteers enter the residence of the person in need of help, the service provider keeps monitoring them using the cameras available at the residence of the person and providing them with further instructions whenever necessary.

\section{B. Computer Vision based Emergency Detection}

In practical use of the system, HM develops a normal behavior model of the elder, during a learning or profiling phase, at the launch of the system. This model can be developed when the elder is supervised by a nurse for a short period of time (say a few days). While receiving contextual information from cameras and sound sensors, HM constantly compares the current behavior with the normal behavior using computer vision techniques. The rationale behind the choice of computer vision underlies beneath the fact that computer vision offers cheap, practical and non-invasive solution in a sense that the hardware is cheap, and the user is not required to put on any device.

Computer vision techniques are used to track the occupant in his home environment and an AI system learns the daily life pattern in order to detect any abnormalities such as falls or unusual activity/inactivity patterns that can be attributed to health problem. In case an abnormal pattern is detected, HM sends an alert to SC. Moreover, computer vision can be used to collect more in-depth information in order for the system to build more accurate models of the user environment and help SC agents to look retrospectively to events leading to any unusual event such as a fall. Here we describe a system we developed for automatically tracking a single occupant in his 
home environment (sitting room) and annotating his activities and detecting any abnormal inactivity which might be a fall [27], [29], [30].

First the occupant is tracked for an extended period of time for HM to learn the usual entry/exit and inactivity zones in the sitting room. Once the learning is done, HM is able to detect any unusual inactivity which can indicate a matter of concern such as a fall and can be used as part of an alarm to alert SC. In this section, we will present the system in more details.

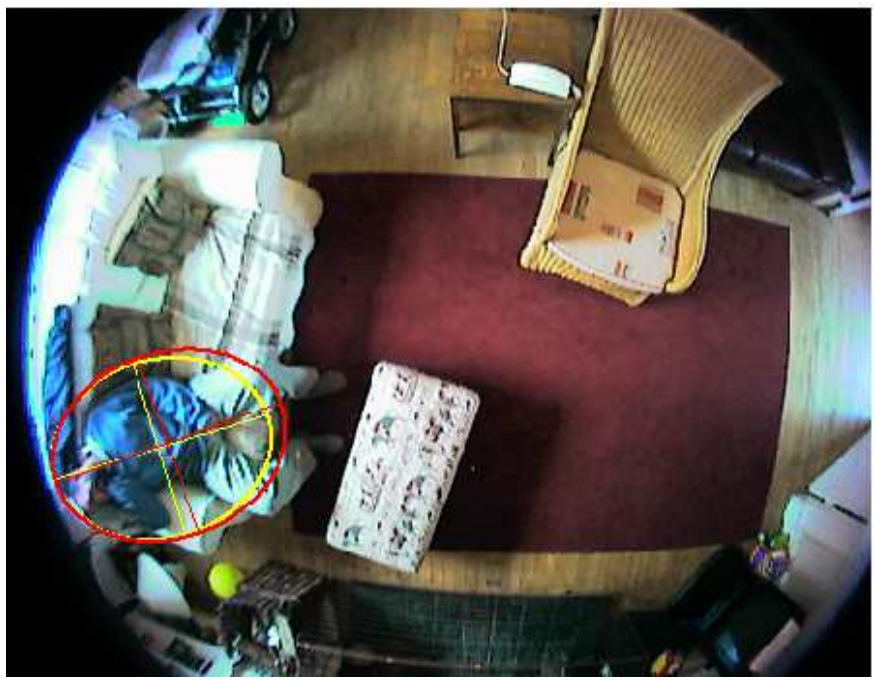

Fig. 8. An example of ellipse estimate (the inner ellipse) obtained by the tracking system.

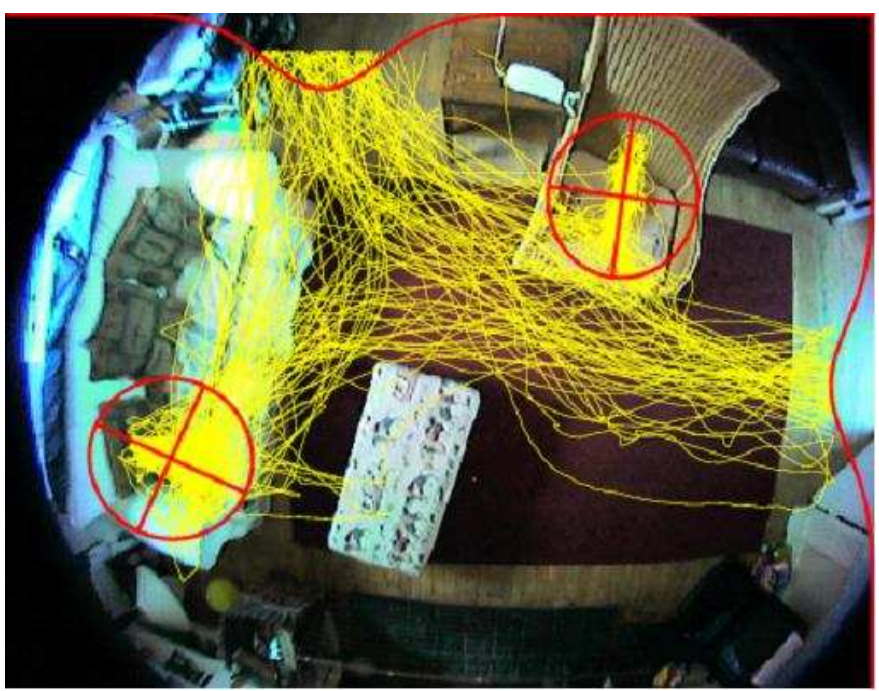

Fig. 9. Smoothed trajectories and example results for learning inactivity zones.

1) Experimental Setup: The layout of the living room can be seen in Fig. 8. The occupant might sit on the sofa to watch television or on the chair to use a telephone. The sofa and the chair are referred to as normal inactivity zones to indicate that the occupant when sitting tends to have little global motion. The room had two entrances which also serve as exits. In [27], physical doorways used to enter the room were not entirely within a camera's field of view and that is why the entries

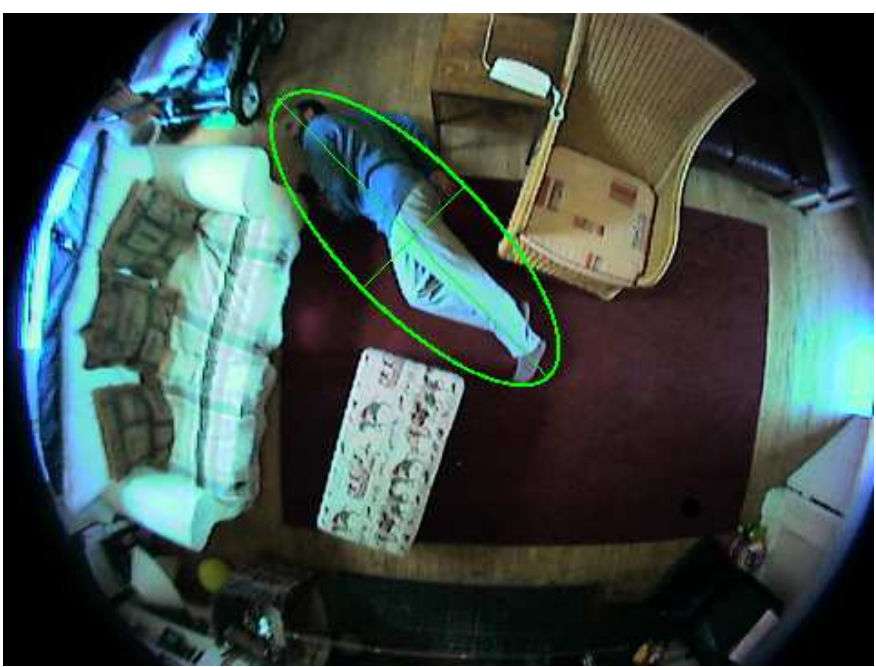

Fig. 10. An example of fall where HM detects the occupant being inactive in an unusual location.

and exits were detected using computer vision. However in our integrated system ANGELAH, the entries and exits were detected via RFID readers.

In the scenario studied in [27], an actor (fourth author) was instructed to perform a series of activities in the room designed to emulate aspects of the way an elder might use such a room. He was instructed to enter the room, visit and stay for some time in one or more inactivity zones (i.e., chair or sofa), then exit the room. In an example, he was instructed to enter through the hall door, sit on the chair and use the telephone, go and sit on the sofa and then exit through the hall door. In addition, the actor was instructed to act few falls in different places in the room to enable evaluation of unusual inactivity. The sequences were acquired using a digital video camera at 30 frames per second over two days of changeable lighting conditions as a result of changing weather.

2) Overhead Monitoring: As the clothing and body postures were highly variable, the person's position, and a shape representation and orientation in the image plane were tracked using an ellipse. A background subtraction technique is used to segment moving objects (i.e., the occupant) from the static background. Then a particle filtering method called Iterated Likelihood Weighting (ILW) [31] with image evidence provided using background subtraction in conjunction with an adaptive background model with shadow detection. Fig. 8 shows a typical estimate of the occupant whereabouts obtained during tracking. The tracker only lost lock in 3 sequences out of all 96 recorded sequences.

3) Context Learning and Unusual Inactivity Detection: The tracker produces temporally discretised, smoothed 2D trajectories in the image plane. When the speed drops below 25 pixels per frame, the occupant is considered inactive. Gaussian mixture models (GMMs) were used to cluster the usual inactivity points. To obtain a Gaussian mixture with components that correspond directly to inactivity zones, penalized likelihood functions that encode priors on cluster scale and shape were incorporated in the learning process. Fig. 9 shows smoothed trajectories and example results for learning 
inactivity zones. The learned model of spatial context were used to temporally segment trajectories and to detect unusual inactivity. When the person's speed drops below 25 pixels per frame, he is considered inactive, and the inactivity zone probability density functions (pdfs) are used to check whether the inactivity occurred in a usual or unusual location. As the speed at each time-step was estimated using a nite difference over a 40-frame temporal window, an inactivity is detected with a delay $\tau_{d}=1.6$ seconds. Fig. 10 shows an example of fall where HM detects that the occupant is inactive in an unusual location.

\section{Collaboration Management}

Upon reception of a SOS message as a result of an emergency detection by an elder's HM, SC promotes the formation of a new group composed of individuals currently located in physical proximity with the elder. Responders are then invited to join the response group.

Similar in spirit to our previous research [20], we implemented a prototype user interface that prevents bystander apathy effect, which may inhibit responders from providing assistance to the elder. The designed application exploits alternative alerting mechanisms to render bystander intervention prompt (Fig. 11). When available LRs receive a CFA message, the application installed on their devices requires users to explicitly acknowledge the received message. The application continuously makes the aware of the emergency notification by emitting a sound signal with an increasing volume till the user accepts or declines the request. ANGELAH exploits acknowledgments from LRs to build a list of volunteers willing to help. In addition, the application user interface provides a speech-based support built on top of IBM Via-Voice that enables responders hand-free interaction with their devices. Fig. 11 portrays the implemented application user interface that provides responders with all necessary information to secure prompt and smooth help to the elder, including password to access the elder's door-lock, "what-to-do" and "howto-do" lists pertaining to tasks that need to be performed. Each task entry in the "what-to-do" list is associated with an entry into the "how-to-do" list that briefly instructs users, untrained or unfamiliar with the system, on how to perform the corresponding tasks.

ANGELAH introduces different forms of overhead depending on the underlying management functions, such as group creation and context-dependent view dissemination. To evaluate the performance of ANGELAH, three critical quantifying parameters are envisioned:

- ANGELAH's responsiveness in creating the elder support groups: defined as the amount of time required for forming an elder support group. It should be emphasized that prompt assistance is of utmost importance in in-house safety scenarios, particularly in case of serious pathologies such as heart-attacks or severe domestic injuries.

- Responders' device battery consumption imposed by ANGELAH's functions: the importance of this factor stems from the fact that the battery of users' devices should be efficiently utilized not only to secure a large number of
LRs ready to assist in an emergency situation but also to guarantee their devices' operability during the rescue operations.

- Memory requirements at the user's terminals: this factor is also critical given the fact that ANGELAH's services are expected to run over portable devices, which are characterized by constrained resources.

We have tested ANGELAH responsiveness in scenarios simulated with NS-2 and consisting of a number of devices randomly deployed over the same locality, i.e., the same wireless cell (Fig. 12). To investigate the functionality of ANGELAH under different network conditions, we range the number of responders from 2 to 100. Our simulation settings consider IEEE 802.11 networks with $10 \mathrm{Mbps}$ transmission rate, random waypoint mobility pattern and speeds ranging from 1 to $3 \mathrm{~m} / \mathrm{s}$. For the sake of simplicity, users are assumed to respond immediately to CFA messages; time needed for a user to detect alerting messages is set to zero. Admittedly, in real-life scenarios, this time may dominate the overall group formation time. The simulations demonstrate that ANGELAH responsiveness is, on average, of few seconds and tend to degrade to few tens seconds when more than 60 LRs are available. Responsiveness degrades due to increase in packet losses, due in turn to wireless network characteristics (e.g., limited bandwidth and channel error rates), in addition to a minimal contribution from the computational load that comes with the profile repository fetching the profiles of users and with ERDM solving the MADM problem for the selection of adequate volunteers.

The battery degradation depends mainly on the group maintenance overhead. In particular, view dissemination is the main factor that contributes to battery degradation. In fact, for group view propagation, VMS requires continuous IEEE 802.11 connections which introduce typically high energy costs. Battery life exhaustion augments when view dissemination rate increases. We tried different trade-offs to ensure that the emergency tasks can be totally completed without exhausting the responders' device battery. We empirically found that the time between consecutive views dissemination should be setup from within the range between 10 to 15 seconds.

Fig. 13 depicts the memory requirements as seen from a single responder device. The figure shows the overall ANGELAH memory requirements over time. In our experiments, we tracked memory use for a responder device in a locality where three other responders were available. The experiment lasted for a duration of 13 minutes. All data were obtained by exploiting the JConsol profiling tool. From Fig. 13-a, the total amount of the used heap memory varies between $0.7 \mathrm{MB}$ and 1.3 MB, with an average value of about $0.9 \mathrm{MB}$. In addition, non-heap memory including data, code and stack reaches a stable value of about $14 \mathrm{MB}$ (Fig. 13-b). From these results, it can be concluded that the ANGELAH group management support can be easily installed over a PDA. 


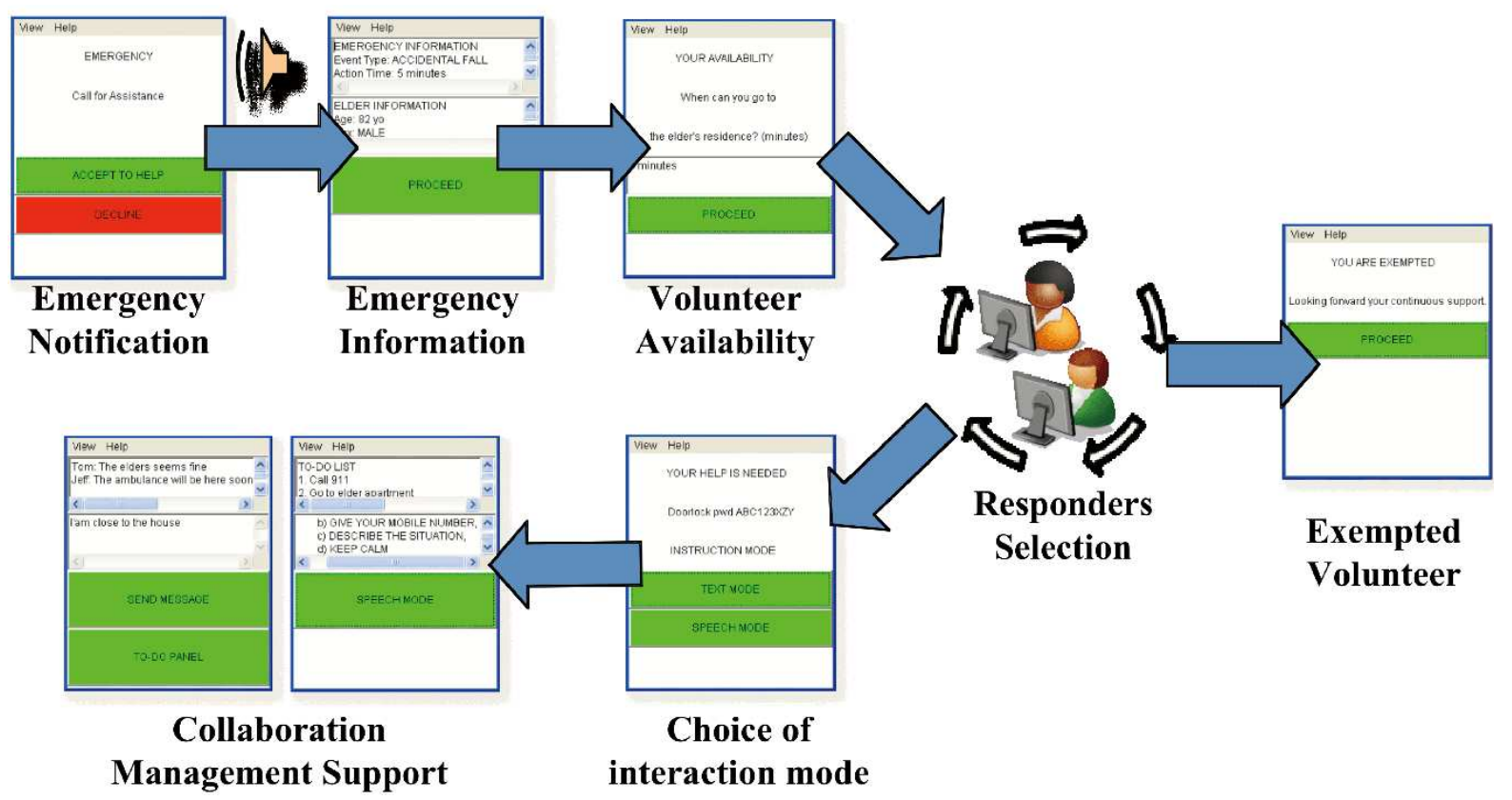

Fig. 11. Responders application user interface.

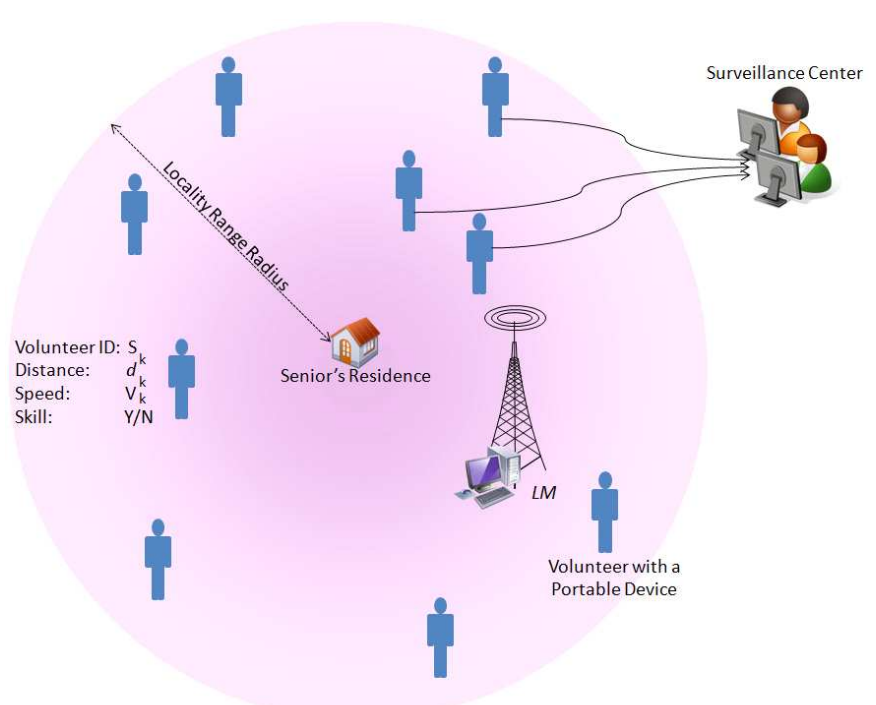

Fig. 12. Simulation scenario.

\section{ANGELAH's Overall Responsiveness}

Admittedly, ANGELAH's overall responsiveness ${ }^{2}$ is an important metric for the system performance evaluation. However, it is highly difficult to exactly determine ANGELAH's responsiveness. This is mainly due to the fact that the system responsiveness depends on unpredictable human factors, such as the behavior of the local responders (e.g., promptness in reacting to CFA messages) and their availability in terms of time which hinges, in turn, on their daily commitments.

In the remainder of this section, we attempt to develop a mathematical analysis that can help in making an estimate

\footnotetext{
${ }^{2}$ Defined as the time elapsed since the emergency occurrence till the arrival of the first helper to the residence of the senior in need of assistance.
}

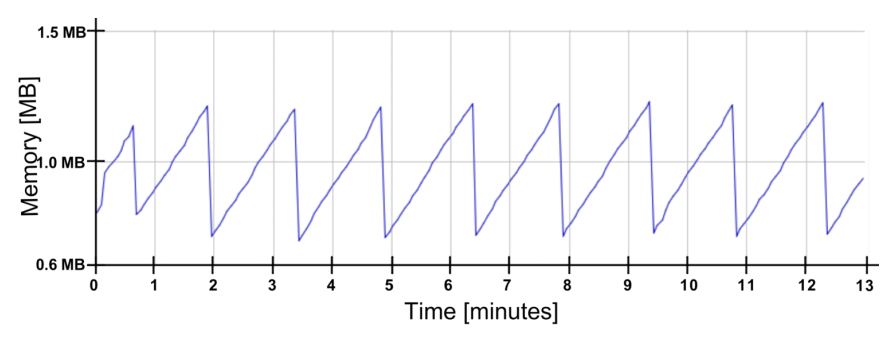

(a) Heap memory.

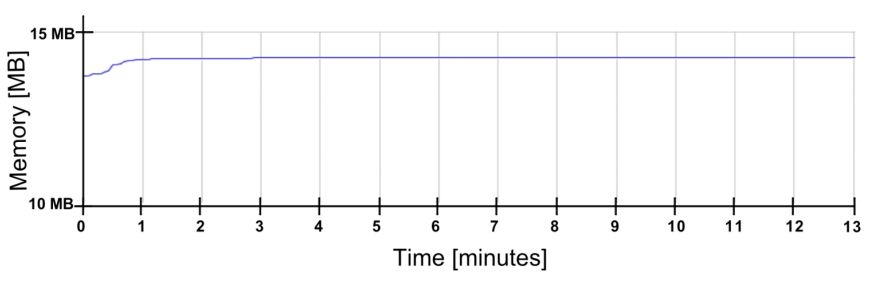

(b) Non-heap memory.

Fig. 13. Memory use on responder devices.

of the system's responsiveness. First, we assume that during the service time, LMs form a statistical profile over time on the number of LRs that visit their locations, whether they are skillful or not, etc. When an emergency event takes place, LMs also keep history track of the responsiveness of LRs; i.e., their reaction to CFA messages via the delivery of AN messages. From this history track, LMs can define a process for the arrival of AN messages at SC. Additionally, from the entire database, LMs can make an estimate on the number of volunteers that may like to assist in an emergency situation occurring at a particular time.

In the following, we attempt to derive the system responsiveness using this information. As a case study, we assume 
that the arrival process of AN messages from LRs follow a Poisson process with arrival rate $\lambda$. Let's consider the case of an emergency level $\Im_{1}$, characterized by the action time $\theta_{\alpha .1}$, the waiting timeout $\tau_{1}$, and the acceptance threshold $\gamma_{1}$. Let also assume that to deal with the emergency level $\Im_{1}$, SC requires at least $N_{1}^{S}$ volunteers with skills or $N_{1}^{T}$ ordinary volunteers (i.e., $\left(N_{1}^{S} \leq N_{1}^{T}\right)$. On the assumption of Poisson process as a process of $\mathrm{AN}$ messages arrival, notification messages from skillful volunteers is also assumed to follow Poisson process with arrival rate $\lambda_{s}\left(\lambda_{s} \leq \lambda\right)$. Based on the same assumption, the mean number of AN messages, from ordinary and skillful volunteers respectively, arriving during the timeout $\tau_{1}$, can be computed as follows:

$$
\begin{gathered}
\beta=\left\lfloor\tau_{1} \cdot \lambda\right\rfloor \\
\beta_{s}=\left\lfloor\tau_{1} \cdot \lambda_{s}\right\rfloor
\end{gathered}
$$

The probabilities of receiving $N_{1}^{T}$ and $N_{1}^{S}$ AN messages from different ordinary and skillful volunteers, respectively, during the timeout $\tau_{1}$ are:

$$
\begin{gathered}
P^{O}=\frac{\left(\tau_{1} \cdot \lambda\right)^{N_{1}^{T}} \cdot e^{-\lambda \tau_{1}}}{N_{1}^{T} !} \\
P^{S}=\frac{\left(\tau_{1} \cdot \lambda_{s}\right)^{N_{1}^{S}} \cdot e^{-\lambda_{s} \tau_{1}}}{N_{1}^{S} !}
\end{gathered}
$$

Again based on the Poisson process assumption of the AN message arrivals, the messages inter-arrival times are mutually independent and identically distributed. We assume these arrivals are separated by $t$ time units and that the first AN message arrives $t$ seconds after the transmission of CFA messages. In case $N_{1}^{T}$ AN messages are received during the timeout $\tau_{1}$, the time required for receiving AN messages from $N_{1}^{T}$ volunteers, $\theta_{O}$ can be computed as follows:

$$
\theta_{O}=N_{1}^{T} \cdot t
$$

On the assumption of Poisson process, the probability density function of $t$ is $f(t)=\lambda e^{-\lambda t}$. Hence, the average value of $\theta_{O}$ is:

$$
\theta_{O}^{a v g}=N_{1}^{T} \cdot \int_{t=0}^{\tau_{1}} \lambda t \cdot e^{-\lambda t} d t
$$

Considering the case when less than $N_{1}^{T}$ AN messages are received within the timeout $\tau_{1}$, the average value of $\theta_{O}$ can be expressed as:

$$
\theta_{O}^{a v g}=\left(1-P^{O}\right) \tau_{1}+P^{O} \cdot N_{1}^{T} \cdot \int_{t=0}^{\tau_{1}} \lambda t \cdot e^{-\lambda t} d t
$$

Similarly, the time required for receiving AN messages from $N_{1}^{S}$ skillful volunteers, $\theta_{S}$ can be computed as follows:

$$
\theta_{S}^{a v g}=\left(1-P^{S}\right) \tau_{1}+P^{S} \cdot N_{1}^{S} \cdot \int_{t=0}^{\tau_{1}} \lambda_{s} t \cdot e^{-\lambda_{s} t} d t
$$

Hence the time required for receiving AN messages since the transmission of CFA messages till entering the volunteer selection procedure, $\theta_{A N}$ is

$$
\theta_{A N}=\max \left(\theta_{O}^{a v g}, \theta_{S}^{a v g}\right)
$$

On the other hand, out of the arriving $N_{1}^{T}$ volunteers, only those that can meet the action time limitation $\theta_{\alpha .1}$ and the acceptance threshold $\gamma_{1}$ that will be selected. If such volunteers exist, the system response time, denoted as $\theta_{\text {all }}$ can be expressed as:

$\theta_{\text {all }}=\theta_{d}+\theta_{S o S}+\theta_{H}+\theta_{A N}+\theta_{E R D M}+\theta_{E}+\min \left(\Delta_{k}\right)$

Here, it should be noted that apart of $\min \left(\Delta_{k}\right)$ that can be estimated from the statistical profile of LRs available at LMs, all other terms can be estimated from SC.

\section{CONCLUSION}

In this paper, we devised a middleware, dubbed ANGELAH, for supporting elders at home. Issues pertaining to elder support group formation were discussed and adequate solutions were proposed. A case study of the ANGELAH framework was envisioned for elders with severe vision impairments. In the case study, sensors and actuators are connected to a central unit, acting as home network manager, able of gathering and aggregating row information from sensing sources and detecting possibly dangerous situations based on computer vision. A prototype user interface was also developed for responders' PDAs. The performance of ANGELAH in the case study was evaluated based on both computer simulations and real-network experiments. Encouraging results were obtained in terms of battery consumption and memory use at helpers' terminals. A mathematical analysis was also developed for the system's overall responsiveness.

We are aware that the success of our prototype deeply hinges on several aspects, ranging from socio-psychological and aesthetic to technical considerations. Our concern is to stimulate further research work in the area with every hope to see robust systems, based on an efficient integration of both computing and telecommunication technologies, which better assist elders at home and leverage their sense of safety and autonomy. The research work, outlined in this paper, can also define interdisciplinary projects where medical experts, caregivers, and computer/network engineers can collaborate. Indeed, as an example, caregivers can produce a library of emergency scenarios that an elder with a particular pathology is likely to encounter. Medical experts can define emergency levels for each scenario, determine critical action times, and list appropriate instructions. Engineers, in turn, can use this information to define mechanisms for support group management and find adequate solutions to related MADM problems.

\section{REFERENCES}

[1] United Nations Population Division, "World Population Prospects: the 2000 Revision", Web Site: http://www.un.org/esa/population/publications/wpp2000/.

[2] R. Cucchiara, A. Prati, R. Vezzani, "A Multi-Camera Vision System for Fall Detection and Alarm Generation" in Expert Systems journal, vol. 24, n. 5, pp. 334-345, 2007.

[3] D. Bottazzi, R. Montanari, and A. Toninelli, "Context-Aware Middleware for Anytime, Anywhere Social Networks," in IEEE Intelligent Systems, Vol. 22, No. 5, Sep./Oct. 2007. pp. 23-32

[4] R.L. Keeney, H. Raiffa, "Decisions with Multiple Objectives: Preferences and Value Trade-offs," Wiley, New York, 1976. Reprinted, Cambridge University Press, 1993.

[5] W. C. Mann, "The aging population and its needs," in IEEE Pervasive Computing Mag., Vol. 3, No. 2, Apr.-Jun 2004. pp. 12-14. 
[6] A. Soomro and D. Cavalcanti, "Opportunities and Challenges in Using WPAN and WLAN Technologies in Medical Environments," in IEEE Commun. Mag., Feb. 2007. pp. 114-122

[7] Y. B. Choi, J. S. Krause, H. Seo, K. E. Capitan, and K. Chung, "Telemedicine in the USA: Standardization through Information Management and Technical Applications," in IEEE Commun. Mag., Vol. 44, No. 4, Apr. 2006

[8] Y. Xiao, X. Shen, B. Sun, and L. Cai, "Security and Privacy in RFID and Applications in Telemedicine," in IEEE Commun. Mag., Vol. 44, No. 4, Apr. 2006.

[9] D. Hodgins, A. Bertsch, N. Post, M. Frischholz, B. Volckaerts, J. Spensley, J.M. Wasikiewicz, H. Higgins, F. von Stetten, and L. Kenney, "Healthy Aims: Developing New Medical Implants and Diagnostic Equipment,” IEEE Pervasive Computing, Vol. 7, No. 1, Jan./Mar. 2008. pp. $14-21$.

[10] C. Y. Poon and Y. Zhang, and S. Bao, "A Novel Biometrics Method to Secure Wireless Body Area Sensor Networks for Telemedicine and M-Health," in IEEE Commun. Mag., Vol. 44, No. 4, Apr. 2006.

[11] S. Garawi, R. S. H. Istepanian, and M. A. Abu-Rgheff, "3G Wireless Communications for Mobile Robotic Tele-Ultrasonography Systems," in IEEE Commun. Mag., Vol. 44, No. 4, Apr. 2006.

[12] F. Marquis-Faulkes, S.J. McKenna, P. Gregor, and A.F. Newell, "Scenario-based drama as a tool for investigating user requirements with application to home monitoring for elderly people," in Proc. 10th Int'l Conf. on Human-Computer Interaction (HCI'03), Crete, Greece, 2003.

[13] A. Mihailidis, B. Carmichael, and J. Boger, "The Use of Computer Vision in an Intelligent Environment to Support Aging-in-Place, Safety, and Independence in the Home," IEEE Trans. Information Technology in Biomedicine, Vol. 8, No. 3, Sep. 2004. pp. 238-247

[14] M.E. Pollack, L. Brown, D. Colbry, C.E. McCarthy, C. Orosz, B. Peintner, S. Ramakrishnane, and I. Tsamardinos, "Autominder: An intelligent cognitive orthotic system for people with memory impairment," in Robotics and Autonomous Systems, Vol. 44, No. 3-4, Sep. 2003. pp 273-282.

[15] B.P. Lo, J.L. Wang, and G.Z. Yang, "From Imaging Networks to Behavior Profiling: Ubiquitous Sensing for Managed Homecare of The. Elderly," in Proc. 3rd Int'l Conf. on Pervasive Computing, Munich, Germany, May 2005.

[16] A.M. Tabar, A. Keshavarz, and H. Aghajan, "Smart Home Care Network using Sensor Fusion and Distributed Vision-based Reasoning," in Proc. 4th ACM Int'l Workshop on Video Surveillance and Sensor Network, Santa Barbara, USA, Nov. 2006.

[17] K. Z. Haigh, L. M. Kiff, J. Myers, V. Guralnik, C. W. Geib, J. Phelps, and T. Wagner, "The Independent Lifestyle Assistant (I.L.S.A.): AI Lessons Learned," in Proc. 16th Innovative Applications of Artificial Intelligence Conf. (IAAI'04), San Jose, CA., Jul. 2004.

[18] F.D. Mola, G. Cabri, N. Muratori, R. Quitadamo, and F. Zambonelli, "The UbiMedic Framework to Support Medical Emergencies by Ubiquitous Computing," in Int'1 Trans. Systems Science \& Applications, Vol. 1, No. 1, 2006. pp. 15-26

[19] C. Pesto, L. Selavo, Y. Chen, J. Ko, J.H. Lim, A. Terzis, A. Watt, J. Jeng, B. Chen, K. Lorincz, and M. Welsh, "Wireless Medical Sensor Networks in Emergency Response: Implementation and Pilot Results," In Proc. 2008 IEEE Int'1 Conf. on Technologies for Homeland Security, Waltham, MA, May 2008.

[20] D. Bottazzi, A. Corradi, and R. Montanari, "Context-Aware Middleware Solutions for Anytime and Anywhere Emergency Assistance to Elderly People," in IEEE Commun. Mag., Vol. 44, No. 4, Apr. 2006. pp. 82-90

[21] A. Mihailidis, G. R. Fernie, and J. C. Barbenel, "The Use of Artificial Intelligence in the Design of an Intelligent Cognitive Orthosis for People with Dementia," in Assistive Technology, Vol. 13, No. 1, 2001. pp. 2339.

[22] M. Morris and J. Lundell, , "Ubiquitous Computing for Cognitive Decline: Findings From Intels Proactive Health Research,” WA: Intel Corp., Seattle, 2003.

[23] P. Bellavista, D. Bottazzi, A. Corradi, and R. Montanari, "Challenges, opportunities and solutions for ubiquitous eldercare," DEIS University of Bologna Technical Report, Oct. 2005.

[24] A. Mihailidis and G. Fernie, "Context-aware Assistive Devices for Older Adults with Dementia," in Gerontechnology, Vol. 2, No. 2, 2002. pp. 173-189.

[25] A. K. Dey, "Understanding and Using Context," in Personal and Ubiquitous Computing Journal, Vol. 5, No. 1, 2001. pp. 4-7.

[26] P. Krishna and D. Husak, "RFID Infrastructure," in IEEE Applications \& Practice, Vol. 45, No. 9, Sep. 2007. pp. 4-10

[27] S. J. McKenna and H. Nait-Charif, "Summarising Contextual Activity and Detecting Unusual Inactivity in a Supportive Home Environment," in Pattern Analysis and Applications, Vol. 7, No. 4, Dec. 2004, pp. 386-401.

[28] K. Pahlavan, F. Akgul, M. Heidari, A. Hatami, J. Elwell, and R. Tingley, "Indoor Geolocation in the Absence of Direct Path," in IEEE Wireless Commun. Mag., Vol. 13, No. 6, Dec. 2006. pp. 50-58

[29] H. Nait-Charif and S. J. McKenna, "Activity Summarisation and Fall Detection in a Supportive Home Environment," in Proc. Int'l Conf. on Pattern Recognition (ICPR), Cambridge, Aug. 2004.

[30] S. J. McKenna and H. Nait-Charif, "Learning Spatial Context from Tracking using Penalized Likelihood Estimation," in Proc. Int'1 Conf. on Pattern Recognition (ICPR), Cambridge, Aug. 2004.

[31] H. Nait-Charif and S. J. McKenna, "Head Tracking and Action Recognition in a Smart Meeting Room," in Proc. 4th IEEE Int'l Workshop on Performance Evaluation of Tracking and Surveillance, Int'l Conf. on Computer Vision Systems (PETS-ICVS), Graz, Austria, pp. 24-31, Mar. 2003. 